\title{
Before the bubble ruptures
}

\author{
Li Shen, ${ }^{1, *}$ Fabian Denner, ${ }^{1}$ Neal Morgan, ${ }^{2}$ Berend van Wachem, ${ }^{1}$ and Daniele Dini ${ }^{1}$ \\ ${ }^{1}$ Department of Mechanical Engineering, Imperial College London, London, SW7 2AZ, United Kingdom \\ ${ }^{2}$ Shell Global Solutions Ltd., Brabazon House, Threapwood Road, Manchester, M22 ORR, United Kingdom
}

(Received 17 July 2017; published 29 September 2017)

\begin{abstract}
This paper is associated with a video winner of a 2016 APS/DFD Gallery of Fluid Motion Award. The original video is available from the Gallery of Fluid Motion, https://doi.org/10.1103/APS.DFD.2016.GFM.V0092
\end{abstract}

DOI: 10.1103/PhysRevFluids.2.090505

The intensely colorful flow patterns on the surface of a soap bubble can mesmerize and provoke fascination among young children and the scientific community alike. Ever since the pioneering work by Newton [1], Plateau [2], Boys [3], and Marangoni [4], the dynamics of a singular soap bubble or as a constituent of a larger foam system have been studied in a wide range of research areas, in particular, topological coarsening dynamics [5], interfacial thin-film fluid mechanics [6], surface chemistry, and long-range molecular dynamics [7].

We present experimental observations of the pattern evolutions which occur naturally on the surface of a soap bubble before its rupture. These nonlinear flow structures, or Marangoni instabilities, arise due to the effects of surface tension gradients induced by the presence of a surfactant solution. The iridescence is a consequence of the light rays being reflected off the outer surface of the soap bubble. Any changes in the pattern formation alters the distance traveled by the light rays and, therefore, determine whether certain wavelengths of light will interfere constructively or destructively, resulting in the spectrum of colors captured by the experiment.

In Figs. 1(a)-1(d), we see the interesting partition of flow structure on the surface of the concave hemispherical soap bubble. To the right-hand side of Fig. 1(a), the flow is gravity-induced coupled with the Marangoni gradient flow in the direction towards the apex of the hemisphere. We note here that the concentric-ring-patterned objects present in the flow correspond to locally concentrated globules of detergent on the surface. Moreover, we observe a curious nucleation process of dark regions (corresponding to film thickness below $100 \mathrm{~nm}$ ) and its subsequent coarsening dynamics on the left-hand side of Fig. 1(a), with its time-dependent evolution outlined in Figs. 1(b)-1(d). The initial circular geometry of the individual nucleation sites can perhaps be attributed to an isotropic diffusional process at work. As we approach rupture, the geometry becomes irregular as anisotropic forces (such as Marangoni convection) become dominant. On a convex hemispherical surface of a soap bubble, the direction of flow is now away from the apex of the hemisphere and a typical picture of dynamic evolution is captured in Fig. 2. We observe a similar but subtly different network of quasispherical patterns to the concave case possibly due to the different quasielastic response that results from the inverted direction of fluid flow. It is therefore of interest to explore the subtle differences in the pattern formation in the concave and the convex cases, the dynamics beyond the black (sub-100 nm) region and study how the varying geometry and fluid flow impacts the quasielastic response on the bubble thin film.

\footnotetext{
${ }^{*}$ Corresponding author: 1.shen14@imperial.ac.uk

Published by the American Physical Society under the terms of the Creative Commons Attribution 4.0 International license. Further distribution of this work must maintain attribution to the author(s) and the published article's title, journal citation, and DOI.
} 


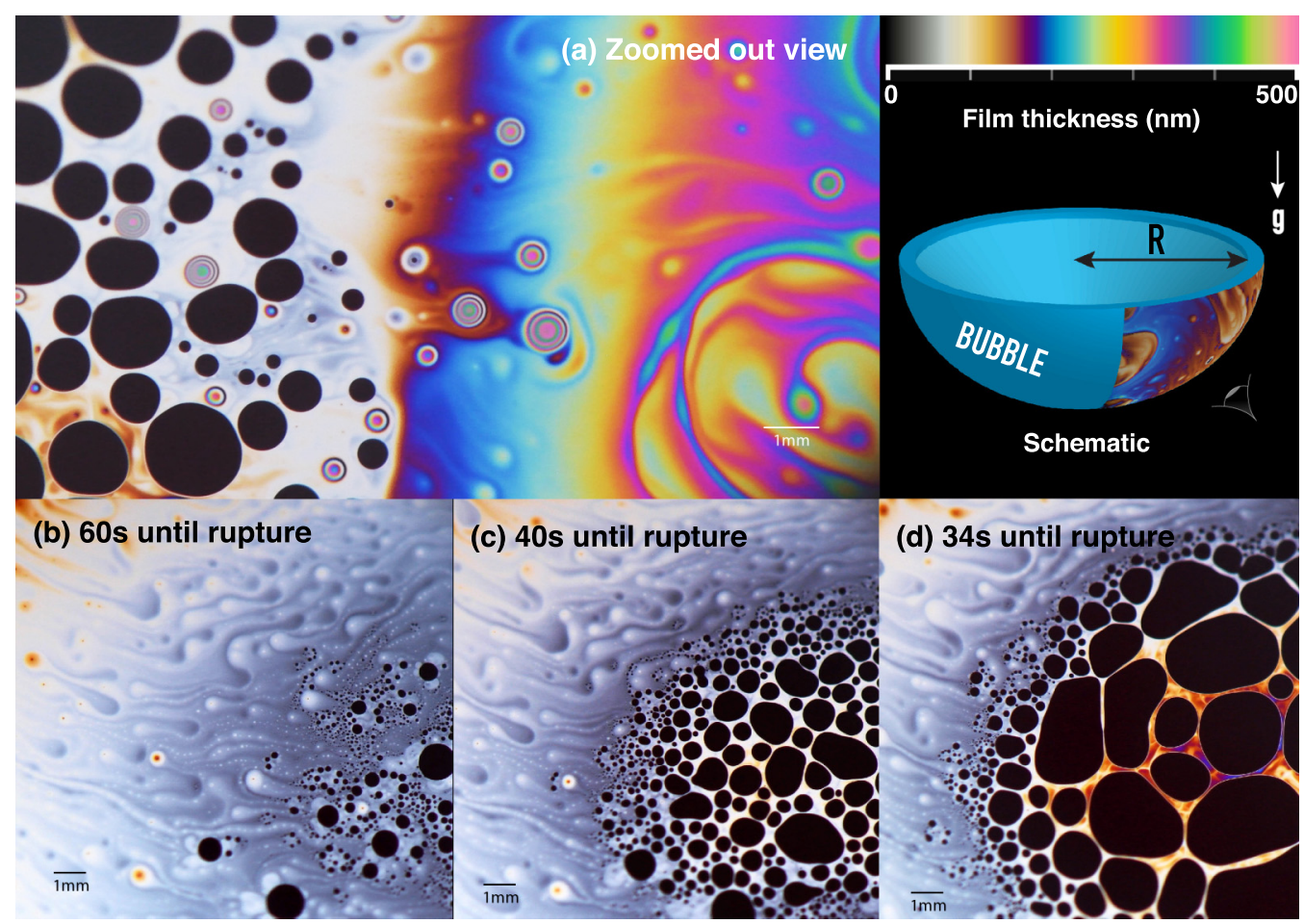

FIG. 1. (a) A section of the concave hemispherical bubble surface. (b)-(d) Details of the two-dimensional von Neumann-like coarsening dynamics in the minute before bubble rupture. The soap bubbles in the experiment have radius $R=2 \mathrm{~cm}$ and are made using a $3 \%$ solution of dishwashing detergent (per volume) and water. DOI: https://doi.org/10.1103/APS.DFD.2016.GFM.V0092

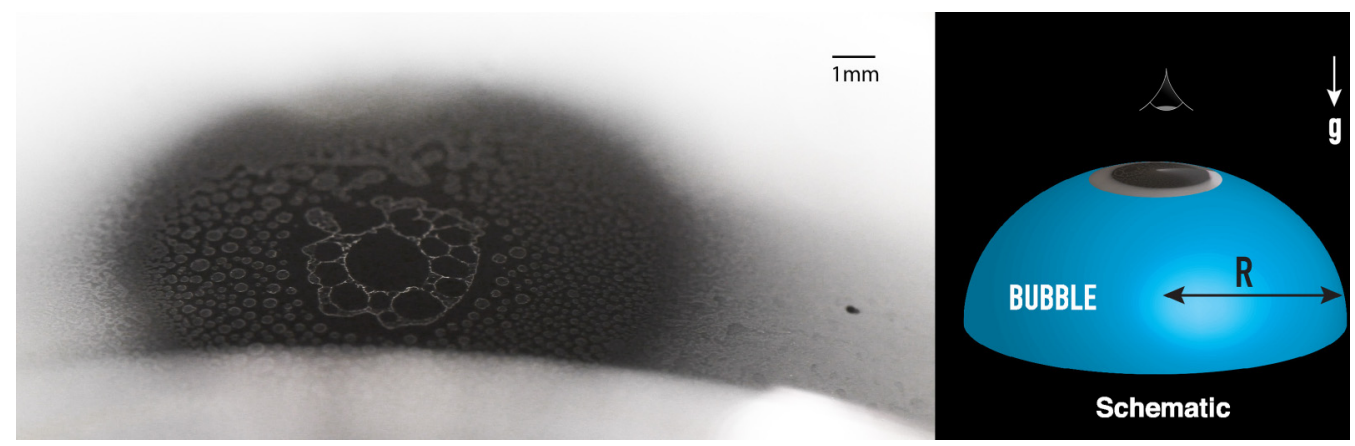

FIG. 2. Pattern formation dynamics of a convex hemispherical bubble configuration before its rupture. The soap bubble is of radius $R=4 \mathrm{~cm}$ also made using a $3 \%$ solution of dishwashing detergent (per volume) and water. DOI: https://doi.org/10.1103/APS.DFD.2016.GFM.V0092 


\section{BEFORE THE BUBBLE RUPTURES}

The authors acknowledge the financial support of the Shell University Technology Centre for fuels and lubricants and the Engineering and Physical Sciences Research Council (EPSRC) through Grants No. EP/M021556/1 and No. EP/N025954/1.

[1] I. Newton, Opticks: A Treatise of the Reflections, Refractions, Inflections and Colours of Light (Dover, New York, 1730).

[2] J. Plateau, Experimental and Theoretical Statics of Liquids Subject to Molecular Forces Only (GauthierVillars, Paris, 1873).

[3] C. V. Boys, Soap Bubbles and the Forces Which Mould Them (Dover, New York, 1896).

[4] G. K. Batchelor, H. K. Moffatt, M. G. Worster, and T. R. Osborn, Perspectives in Fluid Dynamics (Cambridge University, New York, 2003).

[5] R. D. MacPherson and D. J Srolovitz, The von Neumann relation generalized to coarsening of threedimensional microstructures, Nature 446, 1053 (2007).

[6] A. Oron, S. H. Davis, and S. G. Bankoff, Long-scale evolution of thin liquid films, Rev. Mod. Phys. 69, 931 (1997).

[7] P. G. De Gennes, F. Brochard-Wyart, and D. Quéré, Capillarity and Wetting Phenomena: Drops, Bubbles, Pearls, Waves (Springer-Verlag, New York, 2004). 